\title{
Comparación Entre las Velocidades de Calentamiento de un Cautín Inducción Magnética y Otro Compuesto de Resistencia
}

\author{
Ernesto Edgar Mazón Valadez ${ }^{1}$, Alfonso Hernández Sámano ${ }^{1}$, Cuauhtémoc Mazón Valadez ${ }^{1}$, \\ Luis Héctor Quintero Hernández ${ }^{1}$, José Ávila Paz ${ }^{1}$, Mario Eduardo Cano González ${ }^{1}$ \\ 1 Departamento de Ciencias Básicas, Centro Universitario de la Ciéneg, Universidad de Guadalajara, Ocotlán, Jalisco, México.
}

Recibido: 26 Mayo, 2015

Aprobado: 27 Jul., 2015

E-mail: meduardo2001@hotmail.com (MECG)
Resumen: En este trabajo se muestra un análisis comparativo experimental para determinar la diferencia en la velocidad de calentamiento y por lo tanto en el consumo de energía de un cautín de inducción magnética propio y otro tradicional de resistencias de bajo precio, manteniendo una potencia de alimentación constante de $40 \mathrm{~W}$. Se utilizaron tres modelos de puntas comerciales diseñadas para el cautín de resistencia, las cuales son catalogadas por medio de la determinación de sus parámetros magnéticos, para posteriormente determinar su mejor frecuencia de calentamiento con el fin de ajustarla al cautín de inducción, resultando una frecuencia de $100 \mathrm{kHz}$. En general los resultados indican que para cada punta se necesita un menor consumo de potencia usando la inducción magnética, ya que las diferencias entre los tiempos necesarios para fundir el hilo de soldadura van desde el 21,6 al 29,5\%, siendo la punta más ferromagnética aquella que presenta una velocidad de calentamiento más elevada.

Palabras-clave: Fuentes de energía; Flujo de calor; Soldadura.

\section{Comparison Between the Heating Rates of a Magnetic Induction Soldering Iron and Another One Composed of Resistance}

\begin{abstract}
In this work an experimental and comparative analysis is realized in order to determine the difference in the heating rate and thus the power consumption, between a magnetic induction soldering iron and another one of low cost composed of electric resistance. By maintaining a constant power supply $40 \mathrm{~W}$, three models of comercial tips designed for the soldering iron of resistances are used, which are classified by the determination of their magnetic parameters, to subsequently determine the best frequency heating in order to feedback the induction soldering iron, resulting a frequency of $100 \mathrm{kHz}$. Overall the results indicate that for each tip a less power consumption is required using magnetic induction, because the differences between the times required to melt the welding wire ranging from 21.6 to $29.5 \%$, where the tip that exhibited a higher heating speed is the more ferromagnetic.
\end{abstract}

Key-words: Power sources; Heat flux; Solder.

\section{Introdución}

En el mercado de la electrónica existen varias presentaciones de cautines desarrollados especialmente para realizar trabajos técnicos de reparación o desarrollos electrónicos, en los cuales se deben soldar los componentes electrónicos a un circuito impreso por medio de hilo de soldadura de plomo-estaño, o bien, recientemente se cuenta con otras soldaduras ecológicas libres de plomo que pueden componerse de aleaciones de estaño con plata y/o cobre. Estos cautines electrónicos (inventados desde hace varias décadas) se basan en el calentamiento de un elemento térmico a través de una resistencia eléctrica enrollada sobre él, la cual es elaborada de alambre de $\mathrm{NiCr}$, como se muestra en la Figura 1a y ésta se calienta al alimentarla de una corriente alterna. El elemento térmico se encuentra en contacto directo con otra pieza de alta difusividad térmica llamada punta de cautín [1], para transmitir el calor al hilo de soldadura. Aunque en la actualidad también se tienen novedosos dispositivos que se basan en el uso de combustibles fósiles para calentar [2], otros se valen de la generación de corrientes de Eddy por medio de inducción electromagnética [3], como se muestra en la Figura 1b. Estos últimos calientan radialmente sin contacto una parte de la punta del cautín que se encuentra dentro de una bobina, donde se concentran las líneas del campo magnético interactuante de alta frecuencia y posteriormente el calor se difunde hasta el extremo puntiagudo. Como se 
muestra en la Figura 1c la difusión térmica de las puntas tiene una componente radial Dx al estar en contacto con el elemento térmico o con el campo magnético, sin embargo existe otra componente perpendicular Dy para difundir el calor hacia el extremo de la punta.
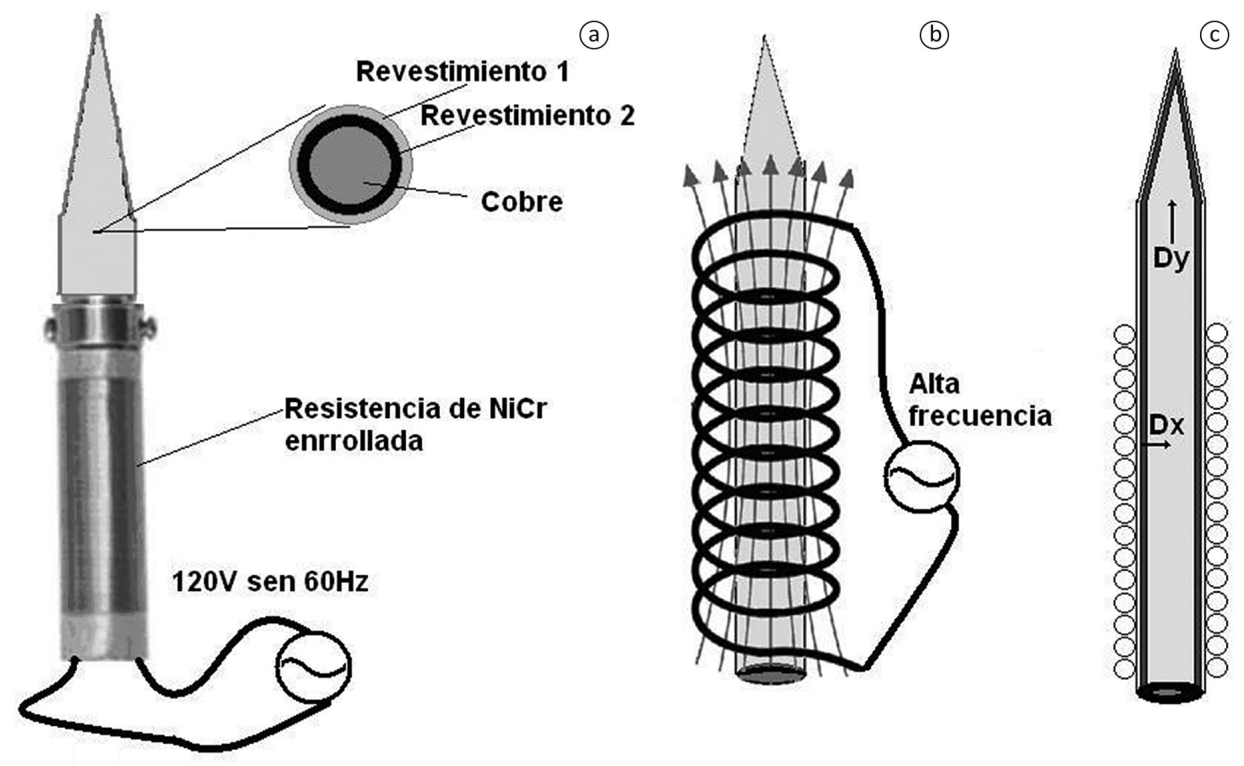

Figura 1. Esquemas del funcionamiento de un cautín (a) de resistencias, (b) de inducción magnética y (c) de las puntas con ilustrando los revestimientos y la difusión térmica. Fuente: Propia.

Por otro lado, las puntas de los cautines de resistencias se disuelven poco a poco con el uso cotidiano [4], típicamente la soldadura de plomo-estaño tiene una temperatura de fusión cercana a los $183^{\circ} \mathrm{C}$, en tanto que las libres de plomo se funden cerca de los $232^{\circ} \mathrm{C}$ [5]. Debido a esta diferencia de temperatura, el uso de la soldadura ecológica ha desencadenado estudios encaminados a la optimización de los procesos de manufactura de las puntas con el fin de alargar su tiempo de disolución [6]. Básicamente éstas se componen principalmente de cobre debido a su alta conductividad térmica [7], sin embargo en su manufactura se observan revestimientos o películas delgadas de otro metal como níquel, cromo, hierro, estaño, como se muestra en las Figuras 2a, c, o bien aleaciones que involucran combinaciones de ellos incluso revestimientos formados por micro-partículas de materiales químicamente inertes [8,9]. Estos revestimientos se usan para incrementar la dureza de la punta y disminuir su corrosión. Adicionalmente, su tiempo de duración se puede incrementar evitando su sobrecalentamiento, lo cual implica que los cautines deben contener una etapa electrónica para sensar su temperatura y retroalimentar una etapa de control electrónico [1,7]. Toda esta electrónica complementaria es necesaria para establecer una temperatura fija controlada que ayuda a realizar trabajos más profesionales, sin embargo se llega a incrementar notablemente el costo de un cautín electrónico de resistencias con estas características.

Asimismo, las puntas de los cautines de inducción magnética (CIM) también deben tener las características ya mencionadas anteriormente de difusividad térmica y baja disolución, sin embargo a esto hay que agregarle la profundidad de penetración de la onda electromagnética s llamado efecto piel [10], que básicamente se trata de una sección efectiva del área transversal del conductor por donde circulan las corrientes inducidas. Este fenómeno depende de la frecuencia $f$ de la onda, de su conductividad eléctrica $\sigma$ (el inverso de su resistividad $\rho=\frac{1}{\sigma}$ ) y de su permeabilidad magnética relativa $\mu$, como se muestra en la Ecuación 1 [11]. A su vez $\mu$ equivale a $(1+\chi)$ donde $\chi$ es la susceptibilidad magnética del material [12].

$$
s=\frac{1}{\sqrt{\pi \sigma \mu f}}
$$



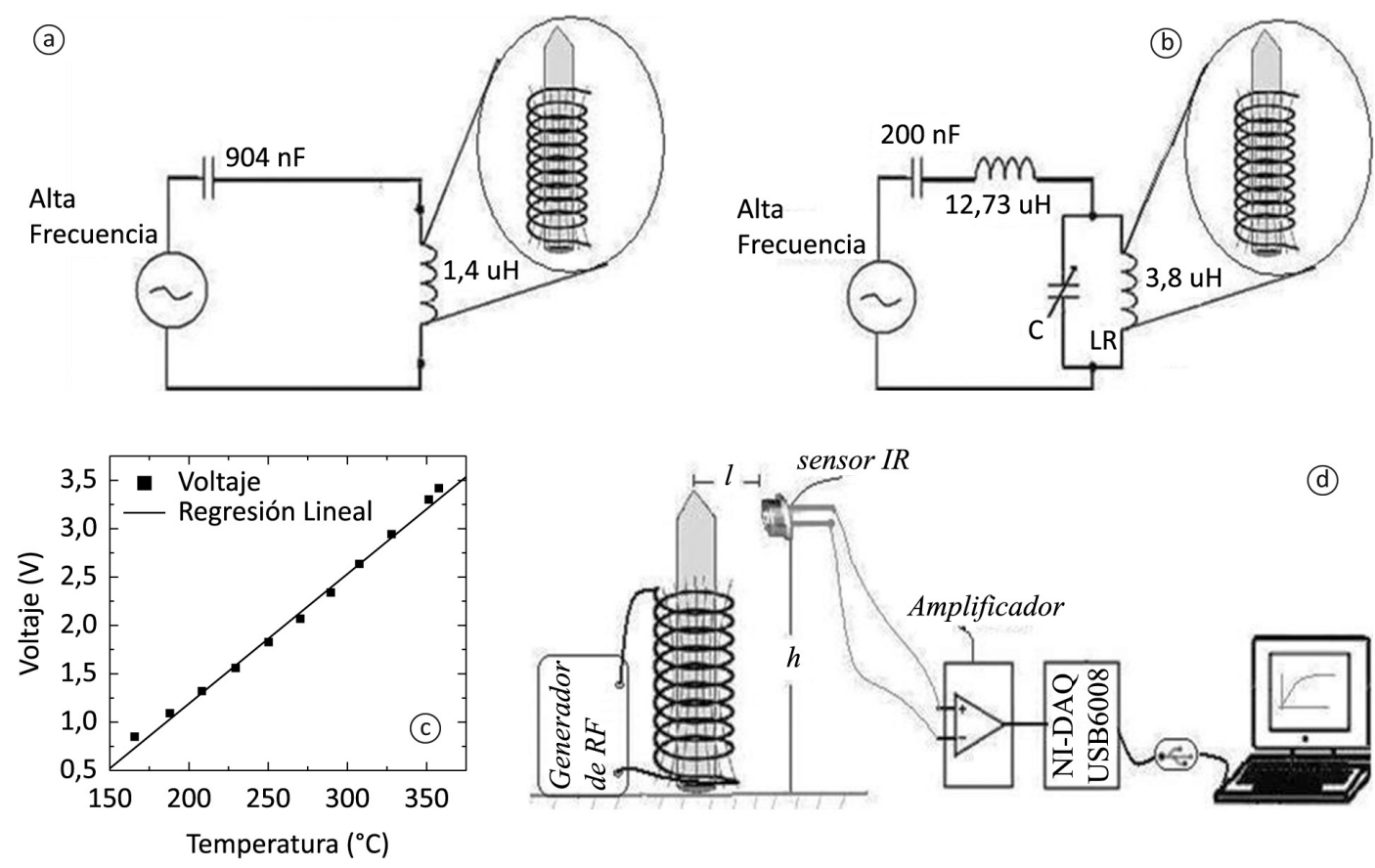

(d)

Figura 2. Esquema del generador de alta frecuencia utilizado y una ampliación de la bobina resonante con la punta (a) a calentar, (b) con la punta caliente sensando su temperatura para graficarla a lo largo del tiempo, (c) regresión lineal para la calibración del sensor de temperatura y (d) esquema del montaje experimental completo. Fuente: Propia.

Es importante estudiar las diversas alternativas que se tienen para soldar dos piezas, en primer lugar para analizar las de uniones con mayor eficiencia, resistencia y dureza [13,14]. O bien para diferenciar las que requieren de un menor consumo de energía. En este sentido, debido a que el calentamiento por inducción magnética se focaliza principalmente en el material a calentar, es posible suponer que un cautín que funcione por medio de este principio físico para calentar directamente su punta, puede llegar a ser más ahorrador de energía que uno de resistencias. No obstante, los CIM están compuestos de un generador de RF, el cual emplea etapas electrónicas que convierten corriente directa en alterna de alta frecuencia [15-17] por lo que pueden llegar a escalar precios elevados en comparación con los de resistencia, misma que sólo se debe alimentar de la tensión doméstica de 50 o $60 \mathrm{~Hz}$ con un simple cable. Por esta razón también resulta atractivo realizar un estudio experimental comparativo del consumo de energía de ambos tipos de cautines para contrastarlo con el precio de producción de cada uno a nivel industrial. Asimismo la industria manufacturera de la electrónica necesita del estudio de estas nuevas tecnologías basadas en la inducción magnética, ya que se supone son más rápidas para calentar o bien necesitan de un menor consumo de energía, todo esto con el fin de optimizar los procesos de manufactura en tiempos y costos.

Se sabe que la potencia disipada por una resistencia y que se tiene que transferir en forma de calor a la punta de diámetro $a$ está dada por la Ecuación 2, donde $i_{R M S}$ es el valor cuadrático medio de la corriente eléctrica y $R$ el valor de la resistencia. En tanto que la potencia disipada en una pieza metálica cilíndrica sólida debido a las corrientes de Eddy se puede aproximar a la Ecuación 3 [18], donde se observa la dependencia del cuadrado de la intensidad magnética $H_{0^{\prime}}$ de la razón entre el diámetro y la penetración $a / s$, o bien de la razón $\rho / s$ denominada resistencia superficial, además existe una contribución no despreciable a su calentamiento debido a cada ciclo de histéresis magnética del material, todo esto indica que se debe escoger una frecuencia apropiada para el tipo de punta a calentar. Un análisis rápido a la Ecuación 3 nos indica que entre más pequeño sea el valor de $s$ por donde circule una cantidad elevada de corriente se disipará más potencia en el conductor. 
Comparación Entre las Velocidades de Calentamiento de un Cautín Inducción Magnética y Otro Compuesto de Resistencia

$$
\begin{aligned}
& P=i_{R M S}^{2} R, \\
& P=\frac{8 \pi H_{0}^{2}}{\sigma} \frac{a}{s}, \text { siempre que } \frac{a}{s}>5 .
\end{aligned}
$$

Mientras tanto, la potencia total $P_{T}$ disipada por un arreglo de $N$ cilindros concéntricos (similar a las puntas con todos sus recubrimientos) de diferentes diámetros $a_{i}$, permeabilidades $\mu_{i}$ y resistividades $\rho_{i}$, expuestas al mismo campo magnético $H$ de frecuencia $f$ con su respectivo $s_{i}$, está dada por la Ecuación 4.

$$
P_{T}=\sum_{i=1}^{N} P_{i}
$$

Recientemente nuestro grupo de investigación ha desarrollado un CIM que opera sin cableado en el mango o soporte [19], el cual ha sido modificado en este trabajo para funcionar con cableado adquiriendo una presentación similar a la de uno de resistencias en forma de lápiz, incluso utilizando el mismo tipo de puntas aunque no sean manufacturadas especialmente para inducción magnética, para este tipo de uso es necesario determinar la mejor frecuencia de calentamiento mediante pruebas en el laboratorio, para sintonizarla al circuito de potencia que compone al CIM. El principal objetivo de este trabajo es realizar una comparación entre la velocidad de calentamiento de dos tipos de cautines diferentes uno de resistencias y nuestro CIM, manteniendo idéntico y constante el consumo de energía de cada uno. Adicionalmente se realiza la comparación usando diferentes tipos de puntas comerciales que son caracterizadas a través de la determinación de sus parámetros magnéticos obtenidos mediante un análisis básico de sus curvas de magnetización.

\section{Materiales y Métodos}

Para iniciar los experimentos se usó un cautín económico de resistencias (US\$ 12 aproximadamente) marca Steren con número de parte CAU-130, de acuerdo con las especificaciones del proveedor éste es capaz de consumir máximo $40 \mathrm{~W}$, alimentado con $127 \mathrm{~V}$ y tiene una resistencia total a temperatura ambiente de $405 \Omega$. Asimismo, para el CAU-130 se adquirieron un par de puntas reemplazables de tres modelos diferentes, las cuales son manufacturadas mayormente de cobre y poseen de geometría cónica. Se eligieron las más económicas y de acuerdo con el proveedor tienen los siguientes números de parte: CAU-118 "de cobre natural" de $67 \mathrm{~mm}$ de longitud y 3,6 mm de diámetro mayor, CAU-192 "de cobre con revestimiento de estaño" de $58 \mathrm{~mm}$ de longitud y 4,5 mm de diámetro mayor y CAU-151 "con revestimiento de hierro-níquel" de $70 \mathrm{~mm}$ de longitud y 3,6 mm de diámetro mayor. Debido a que el proveedor no proporciona información precisa y confiable sobre la manufacturación de las puntas, se realizó una caracterización magnética de las mismas usando un magnetómetro de muestra vibrante Lakeshore 7300 en un rango de intensidad de campo de \pm 9.000 Oe, con el fin de determinar de manera exacta su comportamiento magnético. Para llevar a cabo este análisis se extrajo un trozo cilíndrico de medio centímetro de longitud de cada modelo de puntas y se trazaron sus curvas de magnetización para obtener los parámetros de saturación, remanencia, susceptibilidad y coercitividad magnética.

Por su parte, el CIM que se ha desarrollado consiste en una adaptación del reportado en [19], el cual posee un circuito generador de radiofrecuencias basado en un inversor resonante como en la Figura 2a, donde la bobina que produce la onda electromagnética se encuentra en el lápiz de tal forma que la punta del cautín funge como su núcleo, estando las espiras de cobre y la punta separados por un medio aislante térmico de fibra de vidrio flexible de $3 \mathrm{~mm}$ de espesor, el cual aísla hasta $500{ }^{\circ} \mathrm{C}$. Todas las puntas del CAU-130 se le pueden adaptar al CIM, sin embargo es necesario inicialmente encontrar la mejor frecuencia de calentamiento para cada una de ellas, con el objetivo de ajustar los componentes electrónicos del CIM para operar a esas frecuencias. Con el fin de garantizar un mayor flujo de líneas de campo magnético a través de la superficie de las puntas se mantuvo una bobina tipo solenoide alargada con diámetro interno de $1 \mathrm{~cm}$ y $4 \mathrm{~cm}$ de longitud para introducirlas más de la mitad de su longitud como se esquematiza en las Figuras 1a y 2a. Con esta configuración de la bobina se inducirán corrientes de Eddy en una superficie efectiva de dicha fracción de la punta introducida, donde se focalizará el calentamiento para difundirlo al extremo puntiagudo. 
Debido a que típicamente los recubrimientos más internos de las puntas son películas de hierro o níquel cercanas a los $200 \mu \mathrm{m}$ de espesor, mientras que las más externas de cromo o estaño tienen espesores aproximados a los $10 \mu \mathrm{m}$ [20-22], se han tomado las consideraciones físicas discutidas a continuación. Analizando los valores de las difusividades térmicas $\alpha$ de todos estos materiales mencionados (segunda columna de la Tabla 1) puede observarse que el cobre es algunas veces superior a todos, seguido por el estaño. Sin embargo recordando que la profundidad de penetración $s$ de la onda irradiada (Ecuación 1) depende de sus propiedades electromagnéticas y analizando la tercer columna se observa que las diferencias entre sus resistividades es menor que un orden de magnitud, en tanto que las permeabilidades magnéticas del níquel y el hierro son entre 2 y 3 órdenes de magnitud a mayores a todas las demás (cuarto renglón de la Tabla 1), todo esto indica que para las puntas $\mu$ es el parámetro más predominante en la Ecuación 1 y por lo tanto $s$ en la Ecuación 3. Así que, la principal idea a seguir en este trabajo se basa en calentar con frecuencias tales que las ondas electromagnéticas se enfoquen principalmente en los recubrimientos con mayor $\mu$, es decir $s \approx 200 \mu \mathrm{m}$, para inducir principalmente las corrientes de Eddy en una superficie con mayor resistividad $\rho$ que disipará una mayor potencia, como se explica en la Ecuación 3 , cuyo calentamiento será difundido rápidamente por el cobre hacia la soldadura por su alto valor de $\alpha$. Si se escoge una frecuencia menor a ésta, el valor de $s$ aumentará y se tendrá una menor potencia disipada para difundirse al centro de cobre y por lo tanto una menor velocidad de calentamiento de la punta. Si se escogen frecuencias por encima de los $500 \mathrm{kHz}$ es posible lograr un valor de $s$ pequeño en cada una de las capas conductoras de las puntas, la potencia disipada en el cobre será más significativa que a frecuencias bajas pero aun menor que la disipada en el recubrimiento ferromagnético, de hecho las corrientes de Eddy circularán en una superficie tan delgada del mismo, de tal forma que la difusión térmica Dx de la punta puede ser menor que a frecuencia más bajas. Ahora bien, ya que cada uno de los modelos de puntas empleadas presenta diferencias geométricas no sólo en cuanto a sus longitudes, diámetro y ángulo de cono, sino que también en su composición química (la cual no conocemos de manera precisa), este trabajo se ha enfocado únicamente en analizar las diferencias de calentamiento de una misma punta con las dos técnicas diferentes.

Tabla 1. Propiedades electromagnéticas y difusivas de los materiales.

\begin{tabular}{lccc}
\hline \multicolumn{1}{c}{ Material } & $\mathbf{a}\left(\mathbf{m}^{\mathbf{2}} \mathbf{s}\right) \times \mathbf{1 0}^{-\mathbf{6}}$ & $\boldsymbol{\rho}\left(\mathbf{\Omega}^{*} \mathbf{m}\right) \times \mathbf{1 0}^{-\mathbf{8}}$ & $\boldsymbol{\mu}_{\mathbf{r}}(\mathbf{1})$ \\
Cobre & 111,2 & 1,7 & 1 \\
Níquel & 12,9 & 6,4 & 600 \\
Hierro & 19,3 & 9,7 & 5.000 \\
Estaño & 34,5 & 11,5 & 1 \\
Cromo & 29,1 & 12,5 & 1 \\
\hline
\end{tabular}

Fuente: Propia.

La determinación de las mejores frecuencias de calentamiento se realizó empleando el circuito generador de alta frecuencia reportado en $[15,16]$, al cual se le puede ajustar rápidamente la frecuencia de resonancia modificando su capacitancia resonante Figura $2 \mathrm{~b}$. La metodología utilizada se explica en lo sucesivo: inicialmente se fija una frecuencia $f y$ se introduce una punta en el centro de la bobina resonante, para posteriormente monitorear su temperatura en función del tiempo entre 60 y 70 s, posteriormente se varía la capacitancia resonante para modificar el valor de $f$ siguiendo la aproximación $f=\frac{1}{2 \pi \sqrt{L C}}$, con $L=3,8 \mu \mathrm{H}$ en el generador y se repite el procedimiento hasta barrer un rango considerable de frecuencias, para posteriormente analizar las velocidades de calentamiento de las puntas para cada frecuencia utilizada. Es muy importante verificar que la potencia consumida por el circuito electrónico de alta frecuencia sea siempre la misma para cada valor de $f$. Además, con el fin de evitar errores espurios en la medición de la temperatura por la interacción entre los termómetros metálicos y la radiofrecuencia, esta medición se llevó a cabo usando un sensor de infrarrojo de termo-pila MLX90616 calibrado de manera separada [19]. Es decir, inicialmente se tomó la lectura de voltaje del sensor desde una distancia $I=10 \mathrm{~mm}$ de una pieza metálica que fue calentada a diferentes temperaturas usando una resistencia, al mismo tiempo se tomaron los registros de temperatura usando un termopar clase k conectado al monitor de termopares Stanford Research SR630 y finalmente se realizó la correlación entre ellas por medio de una regresión lineal obteniendo una proporción (pendiente de la curva) de $0,013 \pm 0,004 \mathrm{~V} /{ }^{\circ} \mathrm{C}$, como se puede ver en la gráfica de la Figura $2 \mathrm{c}$. 
Asimismo, en la Figura $2 \mathrm{~d}$ se muestra un esquema del montaje experimental completo, donde se observa el generador de RF que incluye la bobina con la punta incrustada, la cual se encuentra separada una distancia $I$ del sensor de infrarrojo, que a la vez su señal eléctrica es amplificada usando amplificadores operacionales con ganancia total de 7,1 (adimensional), para ser adquirida desde la computadora con una tarjeta de adquisición de datos de National Instruments USB-6008, a través del puerto USB y realizando subrutinas en ambiente Lab-View.

Una vez que se ajustó la frecuencia del CIM, se le suministró una potencia constante $P=40$ W y se procedió a medir su curva de calentamiento para contrastarla con la del CAU-130, usando las mismas puntas y suministrando la misma potencia $P$. Los valores obtenidos de $T$ vs $t$ son almacenados para realizar los análisis posteriormente.

\section{Resultados y Discusión}

Las curvas de magnetización se realizaron a temperatura ambiente y se muestran en la Figura 3a, en todas ellas se observa un comportamiento ferromagnético debido a la presencia de histéresis magnética (área dentro de la curva), que puede observarse mejor en el acercamiento de la Figura 3b. Posteriormente se analizaron cuidadosamente los ciclos de histéresis en los puntos donde las curvas se interceptan con los ejes $M=0$ y $H=0$ para registrar su coercitividad y remanencia magnética respectivamente. Debido a que la susceptibilidad magnética inicial de un material se define como la razón de cambio entre la magnetización contra el campo magnético aplicado en la región lineal, se determinó la susceptibilidad magnética másica inicial de todas las puntas realizando una regresión rectilínea a las curvas en el rango de -300 Oe a 300 Oe, para calcular la pendiente de cada recta ajustada en la plataforma de Origin 6.0.
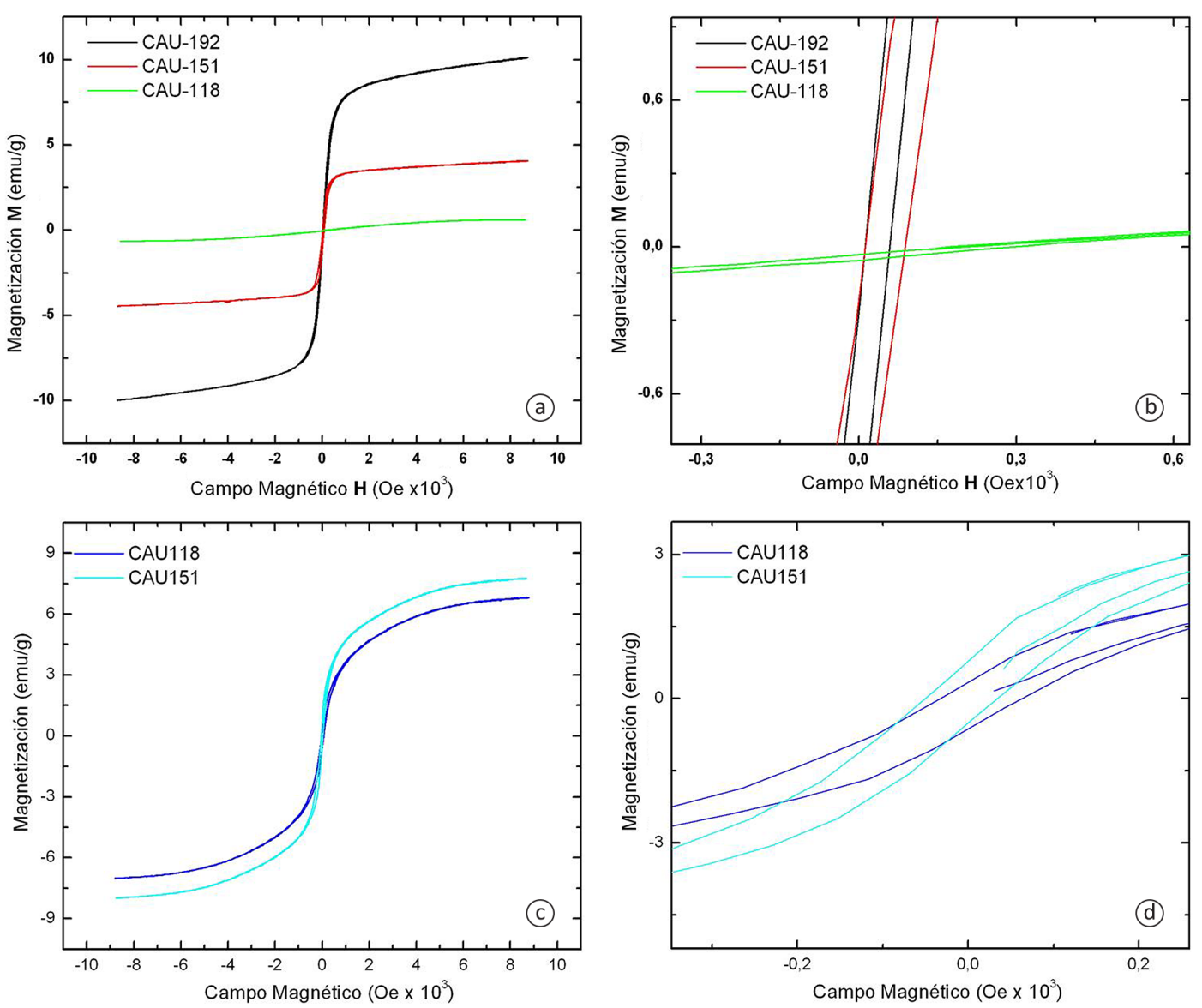

Figura 3. (a) Curvas de magnetización de las puntas CAU-192, CAU-151 y CAU-118, (b) una aproximación para resaltar sus correspondientes ciclos de histéresis, (c) curva de magnetización de un segmento de las puntas CAU-118Ni y CAU-151Ni y su (d) aproximación. Fuente: Propia. 
Los parámetros magnéticos que caracterizan a cada punta se muestran en los tres primeros renglones de datos de la Tabla 2. Claramente se observa que la punta CAU-192 es más magnética que las demás ya que presenta una tendencia a la saturación mayor de aproximadamente $10 \mathrm{emu} / \mathrm{g}$, remanencia de 0,745 emu/g y de la misma forma ocurre con su susceptibilidad magnética inicial $0,23 \mathrm{~cm}^{3} / \mathrm{g}$, pero posee un ciclo de histéresis más cerrado y por lo tanto una menor coercitividad 33,1 Oe, en tanto que la punta CAU-118 es la menos magnética con saturación de $0,6 \mathrm{emu} / \mathrm{g}$, remanencia de $0,042 \mathrm{emu} / \mathrm{g}$ y susceptibilidad de $0,00015 \mathrm{~cm}^{3} / \mathrm{g}$, pero presenta mayor coercitividad 237,5 Oe. Es importante realizar esta caracterización magnética básica ya que las puntas están compuestas en un alto porcentaje de cobre, el cual no ofrece propiedades magnéticas intensas por ser diamagnético, por lo tanto estos parámetros magnéticos se deben principalmente a los recubrimientos anteriormente mencionados y que representan una pequeña fracción de la masa de cada punta.

Tabla 2. Parámetros magnéticos que caracterizan a cada punta.

\begin{tabular}{lcccc}
\hline Tipo de Punta & Coercitividad Hc (Oe) & $\begin{array}{c}\text { Remanencia } \mathbf{M}_{\mathbf{0}} \\
(\mathbf{e m u} \mathbf{g})\end{array}$ & $\begin{array}{c}\text { Susceptibilidad Inicial } \\
\mathbf{X}\left(\mathbf{c m}^{\mathbf{3}} \mathbf{/ g}\right)\end{array}$ & $\begin{array}{c}\text { Saturación }(\mathbf{e m u} \mathbf{g}) \mathbf{a} \\
\mathbf{8 , 5} \mathbf{~ k O e}\end{array}$ \\
CAU-192 & 33,1 & 0,745 & 0,021 & 10,0 \\
CAU-151 & 51,2 & 0,61 & 0,014 & 4,0 \\
CAU-118 & 237,5 & 0,042 & 0,00015 & 0,6 \\
CAU-151Ni & 42,1 & 0,635 & 0,006 & 7,8 \\
CAU-118Ni & 47,5 & 0,475 & 0,006 & 6,9 \\
\hline
\end{tabular}

Fuente: Propia.

Para incrementar el diámetro de las puntas CAU-151 y CAU-118 y sus propiedades magnéticas, se realizó otra pieza cilíndrica hueca usando una placa de níquel de alta pureza de $0,2 \mathrm{~mm}$ de espesor y $40 \mathrm{~mm}$ de largo para revestirlas (denominadas en lo sucesivo CAU-151Ni y CAU-118Ni respectivamente), la curva de magnetización de un trozo de estas dos puntas (similar a los usados en las mediciones de la Figura 3a) incluyendo el revestimiento, se muestran en la Figura 3c. Analizando de nuevo los parámetros magnéticos de ambas curvas en los últimos dos renglones de la Tabla 2, se observa que debido al nuevo revestimiento la primera casi duplicó su saturación magnética en tanto que los otros parámetros se modificaron menos drásticamente, mientras que la segunda modificó abrumadoramente sus propiedades magnéticas y con estas nuevas características se torna más viable su posible calentamiento por inducción. Otra ventaja de usar este recubrimiento de lámina de níquel es que el calentamiento de la punta nunca sobrepasará su temperatura de Curie, es decir los $327^{\circ} \mathrm{C}$ y por lo tanto se pueden omitir etapas de control electrónico para auto-regular su temperatura a fin de evitar su sobrecalentamiento y alargar su tiempo de duración.

Por otro lado, en las Figuras 4a-c se muestran las curvas de calentamientos de cada una de las puntas ( $T$ vs $t$ ) en un rango de frecuencias de $68 \mathrm{kHz}$ a $306 \mathrm{kHz}$, que fueron obtenidas siguiendo el procedimiento mencionado anteriormente y el generador de RF de la Figura $2 \mathrm{~d}$.

Analizando la pendiente de las curvas en una región lineal (de 20 a 40 s) puede observarse en la Figura 4a que la punta CAU-192 se calienta menos rápido a frecuencias en el rango de 68 a $80 \mathrm{kHz}$, en tanto que el calentamiento es más acelerado a los $100 \mathrm{kHz}$. De la misma forma en la Figura $4 \mathrm{~b}$ se observa que la CAU-151Ni se calienta menos en el rango de los 68 a $88 \mathrm{kHz}$, mientras que la velocidad de calentamiento es mayo a los $100 \mathrm{kHz}$. Y finalmente la Figura 4c muestra que la punta CAU-118Ni se calienta más lentamente en el intervalo de 68 a $80 \mathrm{kHz}$ y es más rápido a los $92 \mathrm{kHz}$, además es ligeramente menos rápido a los $100 \mathrm{kHz}$. Por lo tanto se puede concluir debido a estos resultados experimentales que la mejor frecuencia para la CAU-192 y CAU-151Ni es $f=100 \mathrm{kHz}$, sin embargo para la CAU-118Ni la mejor es $f=92 \mathrm{kHz}$. Para simplificar más la construcción de nuestro CIM se eligió una frecuencia única $f=100 \mathrm{kHz}$ sacrificando un $10 \%$ menos la rapidez de calentamiento de la CAU-118Ni. Los valores de $s$ en el cobre, cromo, hierro y níquel son de $0,21,0,17,0,007$ y $0,016 \mathrm{~mm}$ respectivamente, consiguiendo una menor penetración de la onda en los revestimientos ferromagnéticos, que ofrecen una mayor resistividad superficial para incrementar la potencia total disipada $P_{T^{*}}$. 

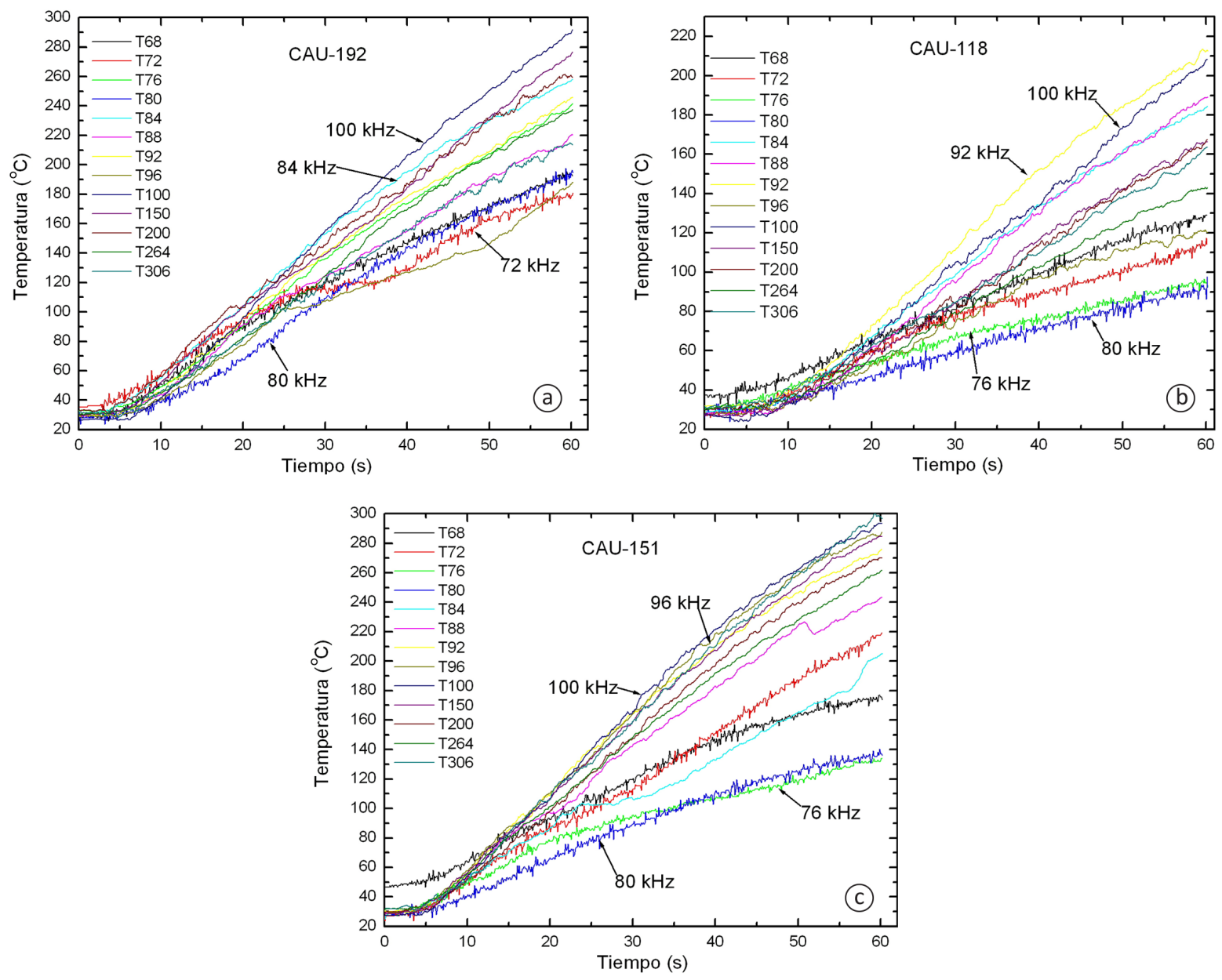

Figura 4. Curvas de calentamiento ( $T$ vs $t$ ) en el rango de frecuencias entre 68 a $306 \mathrm{kHz}$ de las puntas (a) CAU-192, (b) CAU-151Ni y (c) CAU-118Ni. Fuente: Propia.

Después de ajustar las frecuencia de $100 \mathrm{kHz}$ en nuestro CIM se procedió a obtener las curvas de temperatura de cada una de las puntas siguiendo el procedimiento anterior y esto mismo se realizó usando el CAU-130 con las mismas puntas como se muestra en la Figura $5 a$. Después de graficarlos se procedió a realizar una regresión rectilínea a cada curva en su régimen lineal para obtener la pendiente de cada una de ellas. Los resultados de las pendientes obtenidas en unidades de ${ }^{\circ} \mathrm{C} / \mathrm{s}$ se resumen en la Tabla 3, en general se observa una mayor velocidad de calentamiento de las puntas con el CIM en comparación que con el CAU-130 (ver primeros tres renglones de datos), siendo la CAU-192 la más rápida para calentarse, seguida de la CAU-151Ni (20\% más lenta) y la CAU-118Ni es la más lenta de las tres $(27,4 \%)$. La tercera columna de la Tabla 3 indica la diferencia porcentual absoluta de las velocidades de calentamiento de cada punta usando ambos tipos de cautines, de acuerdo con el experimento anterior los tiempos necesarios para lograr la fusión de la soldadura de plomo-estaño con el CIM partiendo de una temperatura ambiente de $30^{\circ} \mathrm{C}$ son de 39,41 y 55 segundos respectivamente. Para alcanzar la fusión de la soldadura libre de plomo los tiempos necesarios son de de 48,50 y 68 segundos, luego se estima que para esas velocidades determinadas los tiempos necesarios para la fusión de ambos tipos de soldaduras usando el CAU-130 incrementan en proporción a las diferencias porcentuales determinadas. En contraste, analizando los últimos dos renglones de la Tabla 3 se observa que al remover la cubierta de lámina de níquel el calentamiento de la CAU-151 y la CAU-118 es mucho mayor con el CAU-130 que con nuestro CIM, lo cual indica que la mayoría de las corrientes de Eddy se generaban en dicho recubrimiento.

Finalmente la Figura 6 muestra una fotografía del CAU-130 y nuestro CIM con su generador de RF de $100 \mathrm{kHz}$, incluyendo las puntas utilizadas con la lámina cilíndrica de níquel empleada como recubrimiento. 

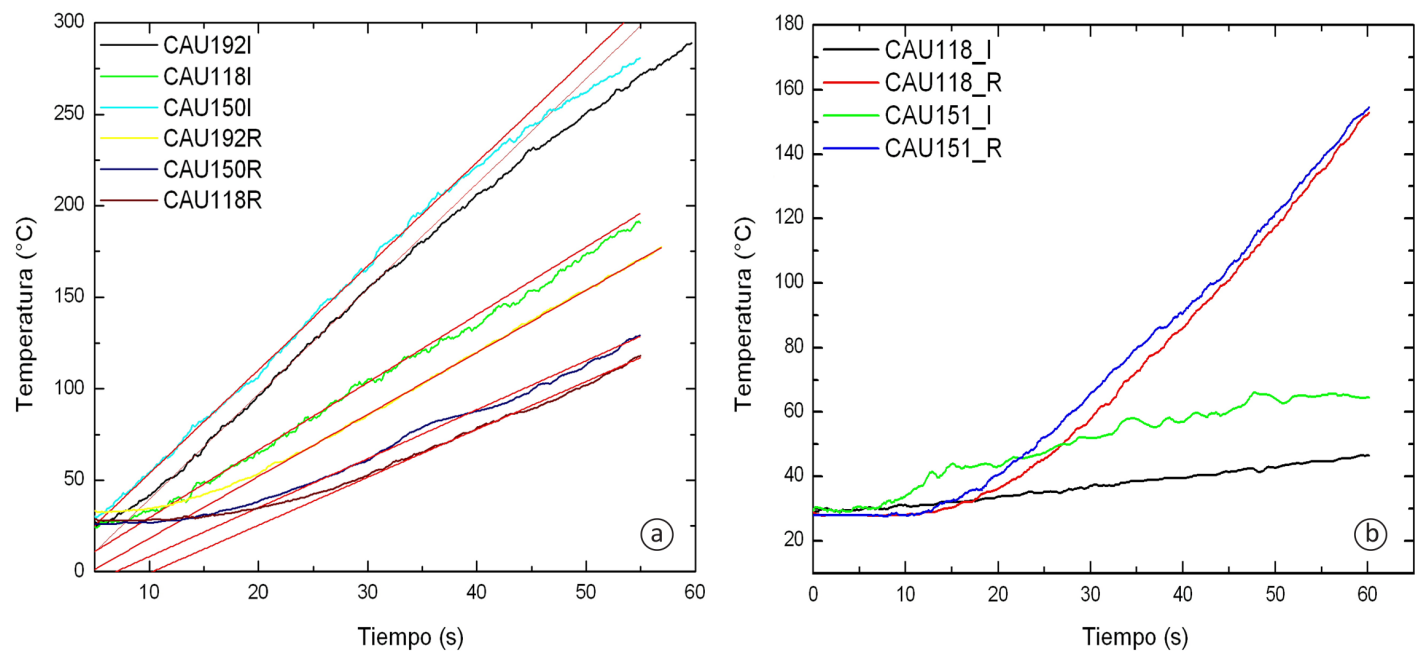

Figura 5. Curvas de calentamiento ( $T$ vs $t$ ) de las puntas CAU-192, CAU-151Ni y CAU-118Ni usando el CIM y el CAU-130 con sus respectivos ajustes lineales y (b) curvas $T$ vs $t$ de las puntas CAU-151 y CAU-118 con ambos cautines. (R significa resistencia, I inducción). Fuente: Propia.

Tabla 3. Velocidades de calentamiento de cada punta usando el CIM y el CAU-130.

\begin{tabular}{lccc}
\hline \multicolumn{1}{c}{ Tipo de Punta } & $\mathbf{v}_{\mathbf{C I M}}\left({ }^{\circ} \mathbf{C} / \mathbf{s}\right)$ & $\mathbf{V}_{\text {CAU-130 }}\left({ }^{\circ} \mathbf{C} / \mathbf{s}\right)$ & $\mid$ Diferencia $\% \mid$ \\
CAU-192 & 5,1 & 4,0 & 21,6 \\
CAU-151Ni & 4,4 & 3,1 & 29,5 \\
CAU-118Ni & 3,7 & 2,8 & 24,3 \\
CAU-151 & 0,7 & 3,1 & 77,4 \\
CAU-118 & 0,3 & 3,0 & 90,0 \\
\hline
\end{tabular}

Fuente: Propia.

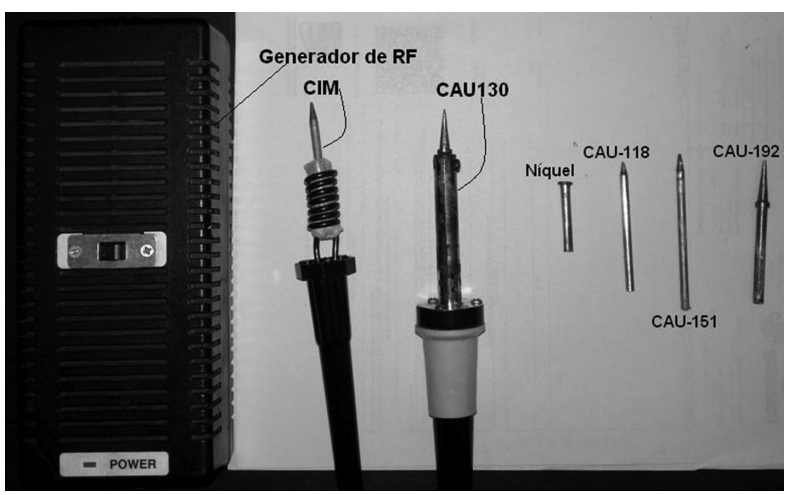

Figura 6. Fotografía del CIM desarrollado con su respectivo generador de RF, del CAU-130, del cilindro de níquel usado como recubrimiento y de todas las puntas utilizadas. Fuente: Propia.

\section{Conclusiones}

En este trabajo experimental se ha realizado la comparación de las velocidades de calentamiento de un cautín tradicional de resistencias de muy bajo precio, contra uno propio que funciona bajo el fenómeno de la inducción magnética, las puntas utilizadas en los experimentos son de muy bajo costo y aunque están especialmente diseñadas para trabajar con cautines de resistencias se ha mostrado que podrían ser usadas para trabajar por medio de inducción magnética. Particularmente dos de ellas fueron recubiertas con una lámina de níquel para incrementar 
Comparación Entre las Velocidades de Calentamiento de un Cautín Inducción Magnética y Otro Compuesto de Resistencia

sus propiedades magnéticas y mejorar drásticamente su respuesta al calentamiento por inducción, en tanto que la CAU-192 no necesita de ninguna modificación.

De acuerdo con las mediciones realizadas manteniendo una potencia constante de 40W, se ha observado que con una onda electromagnética de $100 \mathrm{kHz}$, son más rápidas de calentarse aquellas que presentan propiedades magnéticas más elevadas. En general se ha observado que una misma punta requiere un menor tiempo (y por lo tanto un menor consumo de energía) para alcanzar la temperatura de fusión del hilo de soldadura por medio de inducción magnética que por medio del cautín de resistencias, mostrando diferencias de entre el $21,6 \%$ y $29,5 \%$, es decir que manteniendo un consumo de potencia constante 0,040 kWh usando el CIM se consigue un ahorro de energía en el rango de 0,007 kWh hasta 0,0118 kWh en comparación con el CAU-130. Sin embargo el costo necesario para reproducir el CIM es casi 3 veces el precio del CAU-130, aunque la incorporación del revestimiento externo de lámina de níquel le brinda al CIM un beneficio extra tornándolo en auto-regulable lo que protege a las puntas CAU-118 y CAU151 de sobrecalentamiento debido a su relativamente baja temperatura de Curie.

\section{Agradecimientos}

Los autores agradecemos el apoyo de la institución mexicana CONACYT por las becas otorgadas a los estudiantes que participan en este trabajo

\section{Referencias}

[1] Juvinall JW. inventor. Temperature-controlled soldering iron. United States patent US 2735923. 1956 Feb 21.

[2] Kao J-S. inventor; Rekrow Industrial Inc., cesionario. Device for fastening barrel of gas operated soldering gun. United States patent US 6805275. 2004 Oct 19.

[3] Miyazaki M. inventor; Miyazaki M., cesionario System and method for Induction heating of a soldering iron. United States patent US. 20100258554 A1. 2010 Oct 14.

[4] Takemoto T, Uetani T, Yamazaki M. Dissolution rates of iron plating on soldering iron tips in molten lead-free solders. Soldering \& Surface Mount Technology. 2004;16(3):9-15,.

[5] Harris PG, Chaggar KS. The role of intermetallic compounds in lead-free soldering. Soldering \& Surface Mount Technology. 1998;10(3):38-52,.

[6] Boll SE, Lake JK, Long RC, Ziolkowsky PM. inventores; Lockheed Martin Corporation, cesionario. Soldering iron tip. United States patent US. 6019270 A. 2000 Feb 1.

[7] Forti MS, Gaugler KW, Vivari JA Jr., Wheeler K. inventores; Nordson Corporation, cesionario. Soldering tip, soldering iron, and soldering system. United States patent US. 7699208 B2. $2010 \mathrm{Abr} 20$.

[8] Kayoko Y, Takashi U, Takashi N, Hiroyuki M. inventores. Soldering iron with replaceable tip. European patent EP. 1752018 B1. 2010 Mayo 25.

[9] Dunham PA, Ozpaker HT, Snell J, Mc Call PR. inventores. Soldering tip with wear- and corrosion resistant coating containing dispersed hard particles. Eropean patent EP. 1 685922 A1. 2006 Ene 10.

[10] Dwight HB. A precise method of calculation of skin effect in isolated tubes. Journal of the American Institute of Electrical Engineers. 1923;42(8):827-831.

[11] Fink DG, Beatty HW, editores. Standard handbook for electrical engineers. 11. ed. New York: McGraw Hill; 1978. p. 18-21.

[12] Cano ME, Cordova-Fraga T, Sosa M, Bernal-Alvarado J, Baffa O. Understanding the magnetic susceptibility measurements by using an analytical scale. European Journal of Physics. 2008;29(2):345354. http://dx.doi.org/10.1088/0143-0807/29/2/015.
[13] Cunha TV, Bohórquez CEN. Ultrassom na soldagem a arco uma revisão acerca dos métodos de aplicação e seus efeitos. Soldagem \& Inspeção. 2014;19(3):221-230. http://dx.doi. org/10.1590/0104-9224/SI1903.04.

[14] Souza $\mathrm{Cl}$, Ferraresi VA. Análise comparativa dos processos de soldagem GMAW e FCAW com transferência metálica por curto-circuito na posição horizontal. Soldagem \& Inspeção. 2013;18(3):268-280. http://dx.doi.org/10.1590/S010492242013000300009 .

[15] Cano ME, Cordova T, Hernandez A, Estradaa JC, Knautha P, Lopez $Z$ et al. Experimental setup for magnetic hyperthermia: pilot study. Revista Mexicana de Física S. 2012;58(2):262-265.

[16] Cano ME, Barrera A, Estrada JC, Hernandez A, Cordova T. An induction heater device for studies of magnetic hyperthermia and specific absorption ratio measurements. The Review of Scientific Instruments. 2011;82(11):114904. http://dx.doi. org/10.1063/1.3658818. PMid:22129001.

[17] Pereira Júnior JP, Bracarense AQ. Brasagem por indução de tubos de alumínio utilizados em trocadores de calor. Soldagem \& Inspeção. 2003;8(2):75-83.

[18] Brown GH, Hoyler CN, Bierwirth RA. Theory and applications of the radiofrequency heating. New York: D. Van Nostrand Company; 1947. $30 \mathrm{p}$.

[19] Mazón-Valadez EE, Hernández-Sámano A, Estrada-Gutiérrez JC, Ávila-Paz J, Cano-González ME. Developing a fast cordless soldering iron via induction heating. Dyna. 2014;81(188):166172. http://dx.doi.org/10.15446/dyna.v81n188.41635.

[20] Yoshimura K, Uetani T, Nagase T, Masaki H. inventores; Hakko Corporation, cesionario. Soldering iron with replaceable tip. United States patent US. 8569657. 2013 Oct 29.

[21] Kent GM. inventor; Plato Products Inc., cesionario. Method of manufacturing replaceable soldering iron tips. United States patent US. 3315350 A. 25 Apr. 1967.

[22] Nagase T, Uetani T, Teraoka Y, Naito T, Miyabe M, Sekimori N. inventores; Hakko Corpotation, cesionario. Soldering iron tip and method of manufacturing same. United States patent application US. 10/785,151. 2004 Nov 18. 